\title{
25 Years of Quantum Hall Effect (QHE) \\ A Personal View on the Discovery, Physics and Applications of this Quantum Effect
}

\author{
Klaus vON KLITZING \\ Max-Planck-Institut für Festkörperforschung \\ Heisenbergstr. 1 \\ D-70569 Stuttgart \\ Germany
}

\section{Historical Aspects}

The birthday of the quantum Hall effect (QHE) can be fixed very accurately. It was the night of the 4th to the 5th of February 1980 at around 2 a.m. during an experiment at the High Magnetic Field Laboratory in Grenoble. The research topic included the characterization of the electronic transport of silicon field effect transistors. How can one improve the mobility of these devices? Which scattering processes (surface roughness, interface charges, impurities etc.) dominate the motion of the electrons in the very thin layer of only a few nanometers at the interface between silicon and silicon dioxide? For this research, Dr. Dorda (Siemens AG) and Dr. Pepper (Plessey Company) provided specially designed devices (Hall devices) as shown in Fig.1, which allow direct measurements of the resistivity tensor.

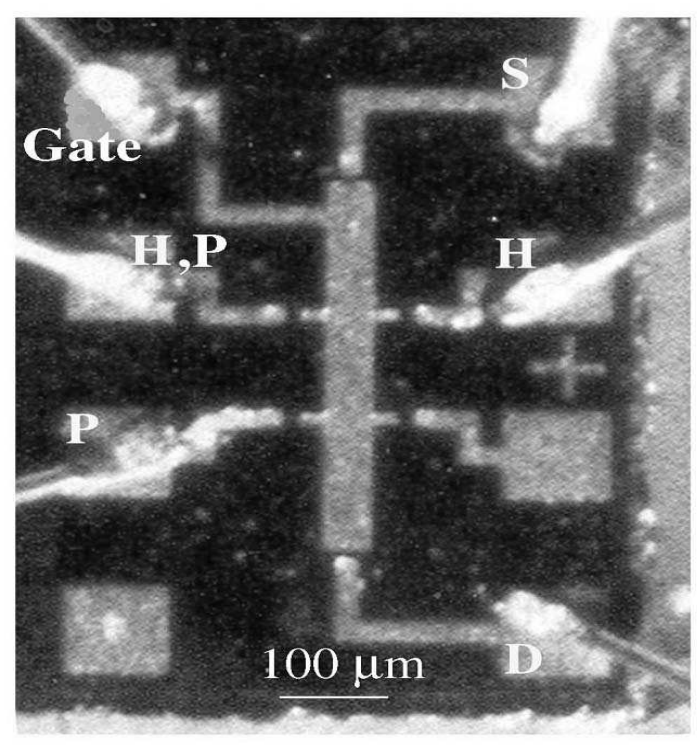

Figure 1: Typical silicon MOSFET device used for measurements of the $x x$ - and $x y$-components of the resistivity tensor. For a fixed source-drain current between the contacts $S$ and $D$, the potential drops between the probes $P-P$ and $H-H$ are directly proportional to the resistivities $\rho_{x x}$ and $\rho_{x y}$. A positive gate voltage increases the carrier density below the gate.

For the experiments, low temperatures (typically $4.2 \mathrm{~K}$ ) were used in order to suppress disturbing scattering processes originating from electron-phonon interactions. The application of a 
strong magnetic field was an established method to get more information about microscopic details of the semiconductor. A review article published in 1982 by T. Ando, A. Fowler, and F. Stern about the electronic properties of two-dimensional systems summarizes nicely the knowledge in this field at the time of the discovery of the QHE [1].

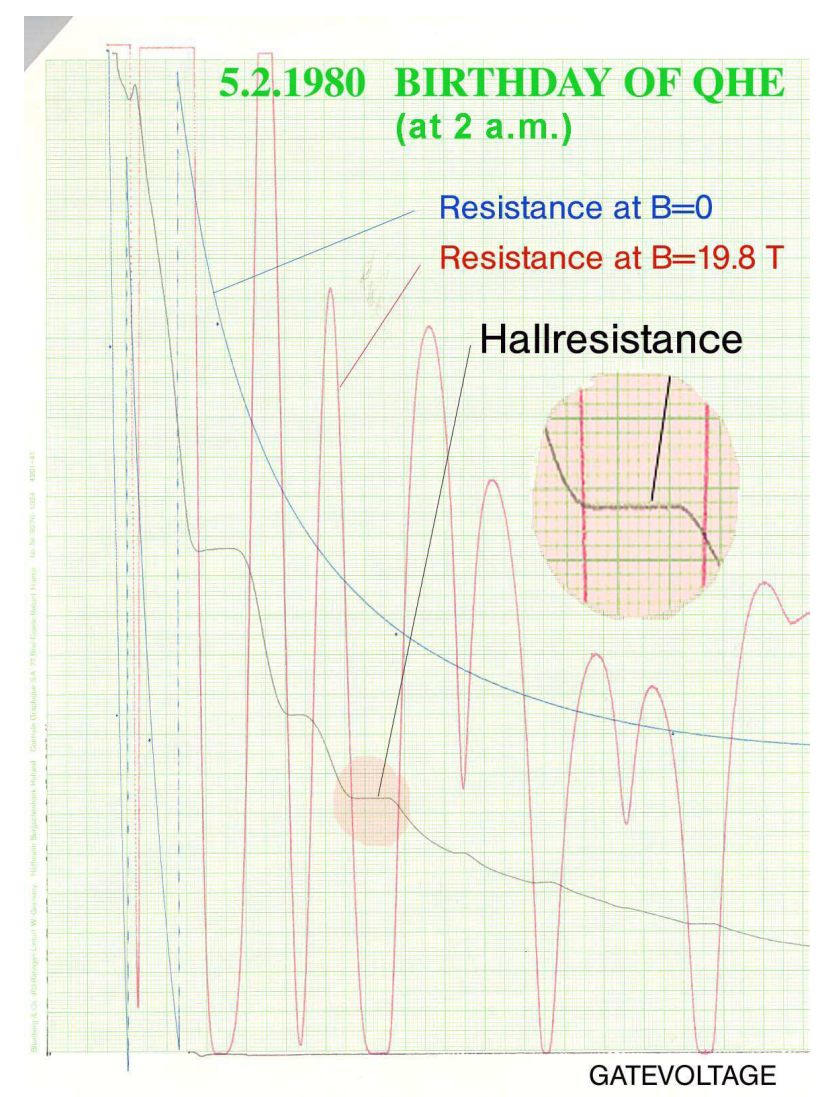

Figure 2: Hall resistance and longitudinal resistance (at zero magnetic field and at $B=19.8$ Tesla) of a silicon MOSFET at liquid helium temperature as a function of the gate voltage. The quantized Hall plateau for filling factor 4 is enlarged.

Since 1966 it was known, that electrons, accumulated at the surface of a silicon single crystal by a positive voltage at the gate (= metal plate parallel to the surface), form a two-dimensional electron gas [2]. The energy of the electrons for a motion perpendicular to the surface is quantized ("particle in a box") and even the free motion of the electrons in the plane of the two-dimensional system becomes quantized (Landau quantization), if a strong magnetic field is applied perpendicular to the plane. In the ideal case, the energy spectrum of a 2DEG in strong magnetic fields consists of discrete energy levels (normally broadened due to impurities) with energy gaps between these levels. The quantum Hall effect is observed, if the Fermi energy is located in the gap of the electronic spectrum and if the temperature is so low, that excitations across the gap are not possible.

The experimental curve, which led to the discovery of the QHE, is shown in Fig. 2. The blue curve is the electrical resistance of the silicon field effect transistor as a function of the gate voltage. Since the electron concentration increases linearly with increasing gate voltage, the electrical resistance becomes monotonically smaller. Also the Hall voltage (if a constant magnetic field of e.g. 19.8 Tesla is applied) decreases with increasing gate voltage, since the Hall voltage is basically inversely proportional to the electron concentration. The black curve shows the Hall resistance, which is the ratio of the Hall voltage divided by the current through the sample. Nice plateaus in the Hall resistance (identical with the transverse resistivity $\rho_{x y}$ ) are observed at gate voltages, where the electrical resistance (which is proportional to the longitudinal resistivity $\rho_{x x}$ ) 


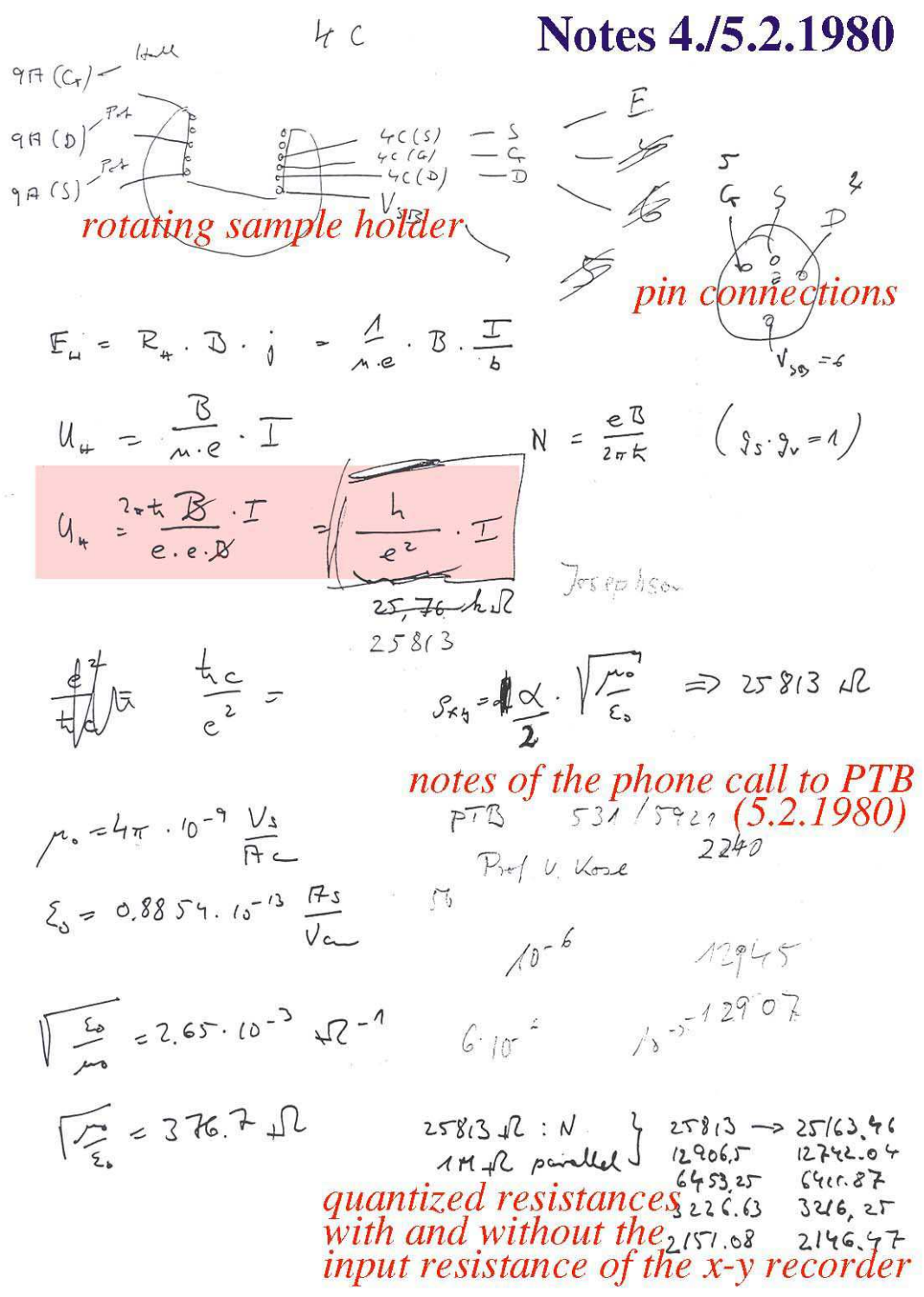

Figure 3: Copy of the original notes, which led to the discovery of the quantum Hall effect. The calculations for the Hall voltage $U_{H}$ for one fully occupied Landau level show, that the Hall resistance $U_{H} / I$ depends exclusively on the fundamental constant $h / e^{2}$.

becomes zero. These zeros are expected for a vanishing density of state of (mobile) electrons at the Fermi energy. The finite gate voltage regions where the resistivities $\rho_{x x}$ and $\rho_{x y}$ remain unchanged indicate, that the gate voltage induced electrons in these regions do not contribute to the electronic transport- they are localized. The role of localized electrons in Hall effect measurements was not clear. The majority of experimentalists believed, that the Hall effect measures only delocalized electrons. This assumption was partly supported by theory [3] and formed the basis of the analysis of QHE data published already in 1977 [4]. These experimental data, available to the public 3 years before the discovery of the quantum Hall effect, contain already all information of this new quantum effect so that everyone had the chance to make a discovery that led to the Nobel Prize in Physics 1985. The unexpected finding in the night of 4./5.2.1980 was the fact, that the plateau values in the Hall resistance $\rho_{x y}$ are not influenced by the amount of localized electrons and can be expressed 
with high precision by the equation $\rho_{x y}=h / i e^{2}$ ( $h=$ Planck constant, $e=$ elementary charge and i the number of fully occupied Landau levels). Also it became clear, that the component $\rho_{x y}$ of the resistivity tensor can be measured directly with a volt- and amperemeter (a fact overlooked by many theoreticians) and that for the plateau values no information about the carrier density, the magnetic field, and the geometry of the device is necessary.

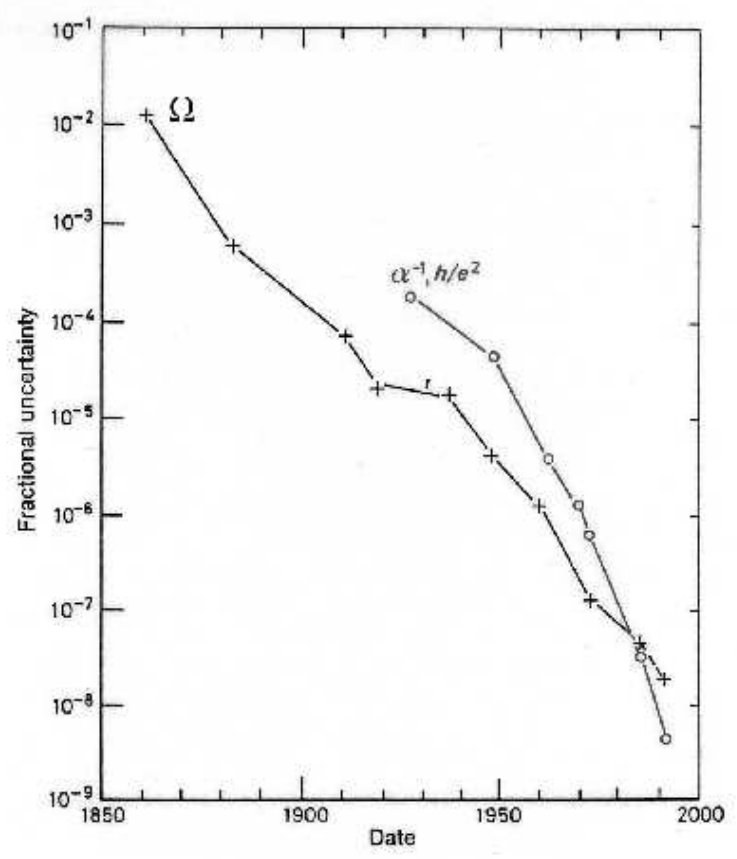

Figure 4: Experimental uncertainties for the realization of the resistance $1 \mathrm{Ohm}$ in SI units and the determination of the fine structure constant $\alpha$ as a function of time.

The most important equation in connection with the quantized Hall resistance, the equation $U_{H}=h / e^{2} \cdot I$, is written down for the first time in my notebook with the date 4.2.1980. A copy of this page is reproduced in Fig. 3. The validity and the experimental confirmation of this fundamental equation was so high that for the experimental determination of the voltage (measured with a $x-y$ recorder) the finite input resistance of $1 M \Omega$ for the $x-y$ recorder had to be included as a correction. The calculations in the lower part of Fig. 3 show, that instead of the theoretical value of 25813 Ohm for the fundamental constant $h / e^{2}$ a value of about $25163 \mathrm{Ohm}$ should be measured with the $x-y$ recorder, which was confirmed with high precision. These first measurements of the quantized Hall resistance showed already, that localized electrons are unimportant and the simple derivation on the basis of an ideal electron system leads to the correct result. It was immediately clear, that an electrical resistance which is independent of the geometry of the sample and insensitive to microscopic details of the material will be important for metrology institutes like NBS in the US (today NIST) or PTB in Germany. So it is not surprising, that discussions with Prof. Kose at the PTB about this new quantum phenomenon started already one day after the discovery of the quantized Hall resistance (see notes in Fig. 3).

The experimental results were submitted to Phys. Rev. Letters with the title: "Realization of a Resistance Standard based on Fundamental Constants" but the referee pointed out, that (at this time) not a more accurate electrical resistor was needed but a better value for the fundamental constant $h / e^{2}$. Interestingly, the constant $h / e^{2}$ is identical with the inverse fine-structure constant $\alpha^{-1}=\left(h / e^{2}\right)\left(2 / \mu_{0} c\right)=137.036 \cdots$ where the magnetic constant $\mu_{0}=4 \pi 10^{-7} N / A^{2}$ and the velocity of light $c=299792458 \mathrm{~m} / \mathrm{s}$ are fixed numbers with no uncertainties. The data in Fig. 4 show indeed, that the uncertainty in the realization of the electrical unit of $1 \Omega$ within the International System of Units (SI units) was smaller (until 1985) than the uncertainty for $h / e^{2}$ 


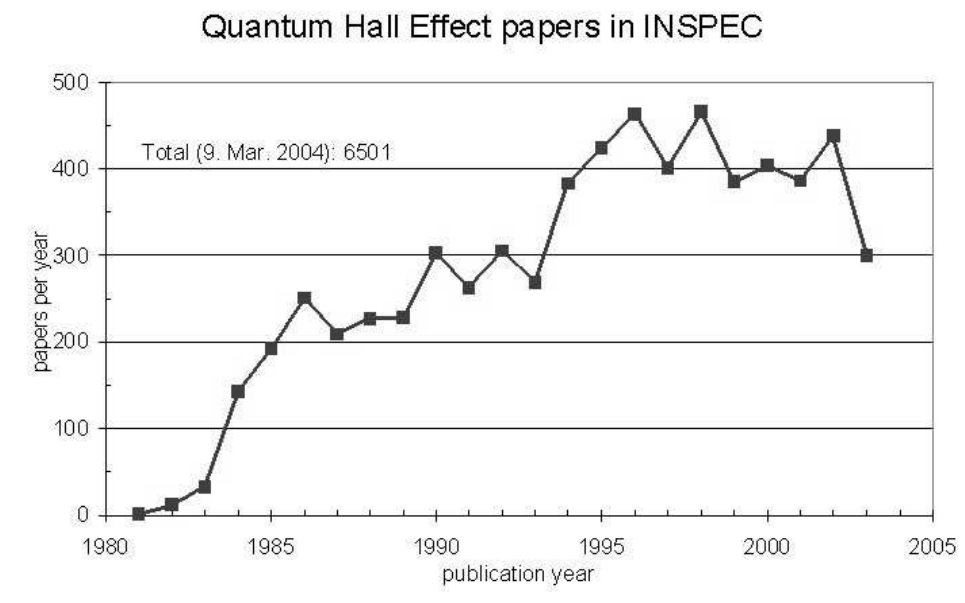

Figure 5: The number of publications related to the quantum Hall effect increased continuously up to a value of about one publication per day since 1995 .

or the inverse fine-structure constant. As a consequence, the title of the first publication about the quantum Hall effect was changed to: "New Method for High-Accuracy Determination of the Fine-Structure Constant Based on Quantized Hall Resistance" [5]. The number of publications with this new topic "quantum Hall effect" in the title or abstract increased drastically in the following years with about one publication per day for the last 10 years as shown in Fig. 5. The publicity of the quantized Hall effect originates from the fact, that not only solid state physics but nearly all other fields in physics have connections to the QHE as exemplarily demonstrated by the following title of publications:

BTZ black hole and quantum Hall effects in the bulk/boundary dynamics [6].

Quantum Hall quarks or short distance physics of quantized Hall fluids [7].

A four-dimensional generalization of the quantum Hall effect [8].

Quantum computation in quantum-Hall systems [9].

Higher-dimensional quantum Hall effect in string theory [10].

Is the quantum Hall effect influenced by the gravitational field? [11].

Up to now, more than 10 books were published about the quantum Hall effect [12-19] and the most interesting aspects are summarized in the Proceedings of the International Symposium "Quantum Hall Effect: Past, Present and Future" [20].

\section{Quantum Hall Effect and Metrology}

The most important aspect of the quantum Hall effect for applications is the fact that the quantized Hall resistance has always a fundamental value of $h / e^{2}=25812.807 \cdots$ Ohm. This value is independent of the material, geometry and microscopic details of the semiconductor. After the discovery of this macroscopic quantum effect many metrological institutes repeated the experiment with much higher accuracy than available in a research laboratory and they confirmed, that this effect is extremely stable and reproducible. Fig. 6 summarizes the data (published until 1988) for the fundamental value of the quantized Hall resistance and it is evident that the uncertainty in the measurements is dominated by the uncertainty in the realization of the SI Ohm. From the internationally accepted definitions for the basic SI units "second", "meter", "kilogram", and "Ampere" it is clear, that all mechanical and electrical quantities are well defined. However the overview in Fig. 7 shows also, that the base unit Ampere has a relatively large uncertainty of about $10^{-6}$ if deduced from the force between current carrying wires. Apparently, the derived unit $1 \Omega=1 \mathrm{~s}^{-3} \mathrm{~m}^{2} \mathrm{kgA}^{-2}$ (which depends in principle on all basic units) should have an even larger uncertainty than $10^{-6}$. However, as shown in Fig. 4, the SI Ohm is known with a smaller uncertainty than the basic unit 


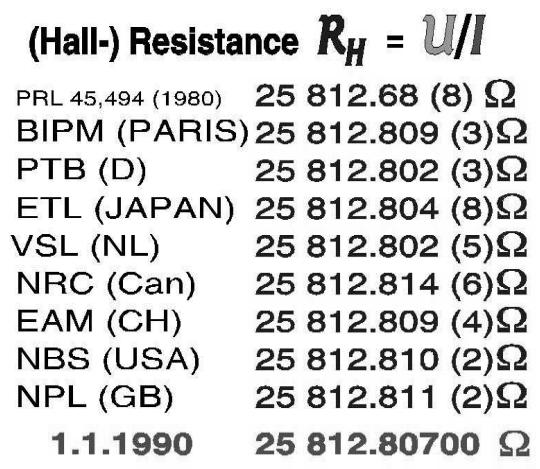

Figure 6: Summary of high precision data for the quantized Hall resistance up to 1988 which led to the fixed value of $25812.807 \mathrm{Ohm}$ recommended as a reference standard for all resistance calibrations after 1.1.1990 .

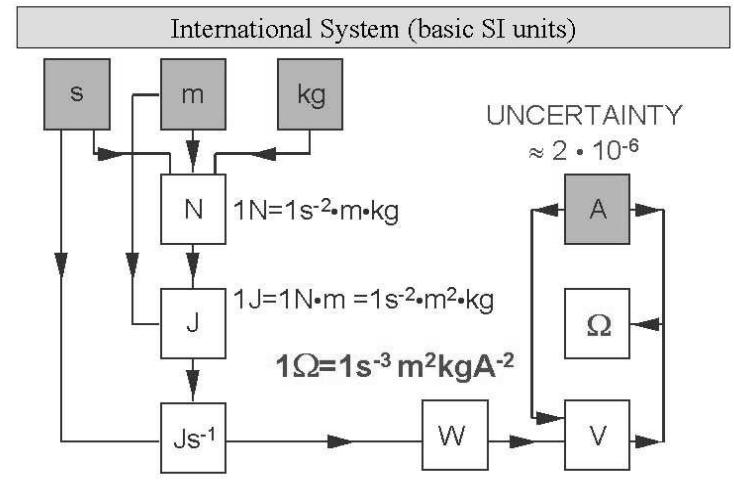

Figure 7: Basic and derived SI units for mechanical and electrical quantities.

Ampere which originates from the fact, that a resistance can be realized via the a.c. resistance $R=1 / \omega C$ of a capacitor $C$. Since the capacitance $C$ of a capacitor depends exclusively on the geometry (with vacuum as a dielectric media), one can realize a SI Ohm just by using the basic units time (for the frequency $\omega / 2 \pi$ ) and length (for a calculable Thomson-Lampert capacitor [21]), which are known with very small uncertainties. Therefore an uncertainty of about $10^{-7}$ for the realization of the SI Ohm is possible so that the fine-structure constant can be measured via the QHE directly with the corresponding accuracy. However, the quantized Hall resistance is more stable and more reproducible than any resistor calibrated in SI units so that the Comité Consultatif d'Electricité recommended, "that exactly $25812.807 \mathrm{Ohm}$ should be adopted as a conventional value, denoted by $R_{K-90}$, for the von Klitzing constant $R_{K}$ " and that this value should be used starting on 1.1.1990 to form laboratory reference standards of resistances all over the world [22]. Direct comparisons between these reference standards at different national laboratories (see Fig. 8) have shown, that deviations smaller than $2 \cdot 10^{-9}$ for the reference standards in different countries are found [23] if the published guidelines for reliable measurements of the quantized Hall resistance are obeyed [24]. Unfortunately, this high reproducibility and stability of the quantized Hall resistance cannot be used to determine the fine-structure constant directly with high accuracy since the value of the quantized Hall resistance in SI units is not known. Only the combination with other experiments like high precision measurements (and calculations) of the anomalous magnetic moment of the electron, gyromagnetic ratio of protons or mass of neutrons lead to a least square 
adjustment of the value of the fine-structure constant with an uncertainty of only $3.3 \cdot 10^{-9}$ resulting in a value for the von Klitzing constant of $R_{K}=25812.807449 \pm 0.000086$ Ohm (CODATA $2002[25]$ ). Accurate values for fundamental constants (especially for the fine-structure constant) are important in connection with the speculation that some fundamental constants may vary with time. Publications about the evidence of cosmological evolution of the fine-structure constant are questioned and could not be confirmed. The variation $\partial \alpha / \partial t$ per year is smaller than $10^{-16}$.

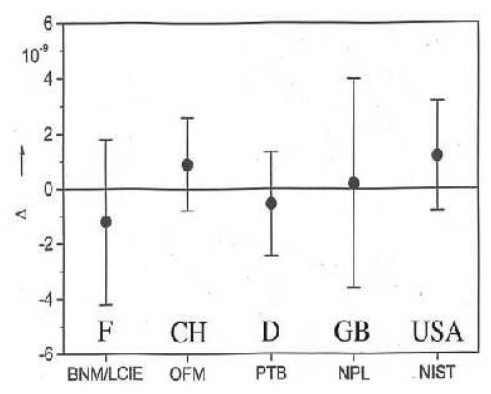

Figure 8: Reproducibility and stability of the quantized Hall resistance deduced from comparisons between different metrological institutes. The observed uncertainties of about $2.10^{-9}$ is two orders of magnitude smaller than the uncertainty in the realization of a resistance calibrated in SI Ohms.

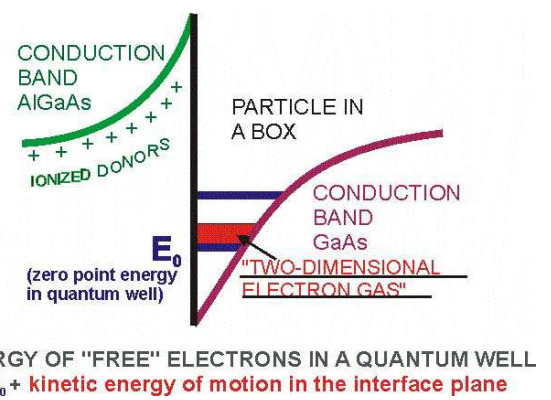

Figure 9: Realization of a two-dimensional electrons gas close to the interface between AlGaAs and GaAs.

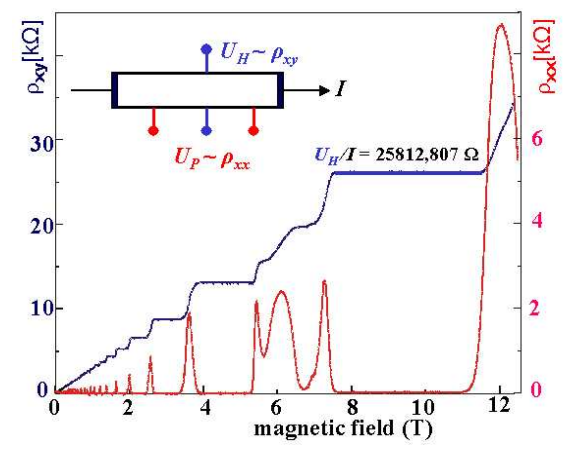

Figure 10: Hall resistance and longitudinal resistivity data as a function of the magnetic field for a GaAs/AlGaAs heterostructures at $1.5 \mathrm{~K}$. 
The combination of the quantum Hall effect with the Josephson effect (which allows an representation of the electrical voltage in units of $h / e$ ) leads to the possibility, to compare electrical power (which depends on the Planck constant $h$ ) with mechanical power (which depends on the mass $m$ ). The best value for the Planck constant is obtained using such a Watt balance [26]. Alternatively, one may fix the Planck constant (like the fixed value for the velocity of light for the definition of the unit of length) in order to have a new realization of the unit of mass.

\section{Physics of Quantum Hall Effect}

The textbook explanation of the QHE is based on the classical Hall effect discovered 125 years ago [27]. A magnetic field perpendicular to the current $I$ in a metallic sample generates a Hall voltage $U_{H}$ perpendicular to both, the magnetic field and the current direction:

$$
U_{H}=(B \cdot I) /(n \cdot e \cdot d)
$$

with the three-dimensional carrier density $n$ and the thickness $d$ of the sample. For a two-dimensional electron gas the product of $n \cdot d$ can be combined as a two-dimensional carrier density $n_{s}$. This leads to a Hall resistance

$$
R_{H}=U_{H} / I=B /\left(n_{s} \cdot e\right)
$$

Such a two-dimensional electron gas can be formed at the semiconductor/insulator interface, for example at the $\mathrm{Si}-\mathrm{SiO}_{2}$ interface of a MOSFET (Metal Oxid Semiconductor Field Effect Transistor) or at the interface of a GaAs - AlGaAs HEMT (High Electron Mobility Transistor) as shown in Fig. 9. In these systems the electrons are confined within a very thin layer of few nanometers so that similar to the problem of "particle in a box" only quantized energies $E_{i}(i=$ $1,2,3 \cdots)$ for the electron motion perpendicular to the interface exist (electric subbands).

A strong magnetic field perpendicular to the two-dimensional layer leads to Landau quantization and therefore to a discrete energy spectrum:

$$
E_{0, N}=E_{0}+(N+1 / 2) \hbar \omega_{c} \quad(N=0,1,2, \ldots)
$$

The cyclotron energy $\hbar \omega_{c}=\hbar e B / m_{c}$ is proportional to the magnetic field $B$ and inversely proportional to the cyclotron mass $m_{c}$ and equal to $1.16 \mathrm{meV}$ at 10 Tesla for a free electron mass $m_{0}$.

Due to the electron spin an additional Zeeman splitting of each Landau level appears which is not explicitly included in the following discussion. More important is the general result, that a discrete energy spectrum with energy gaps exists for an ideal 2DEG in a strong magnetic field and that the degeneracy of each discrete level corresponds to the number of flux quanta $(F \cdot B) /(h / e)$ within the area $F$ of the sample. This corresponds to a carrier density $n_{s}=e \cdot B / h$ for each fully occupied spin-split energy level $E_{0}, N$ and therefore to a Hall resistance $R_{H}=h / i \cdot e^{2}$ for $i$ fully occupied Landau levels as observed in the experiment. A typical magnetoresistance measurement on a GaAs/AlGaAs heterostructure under QHE conditions is shown in Fig. 10. 


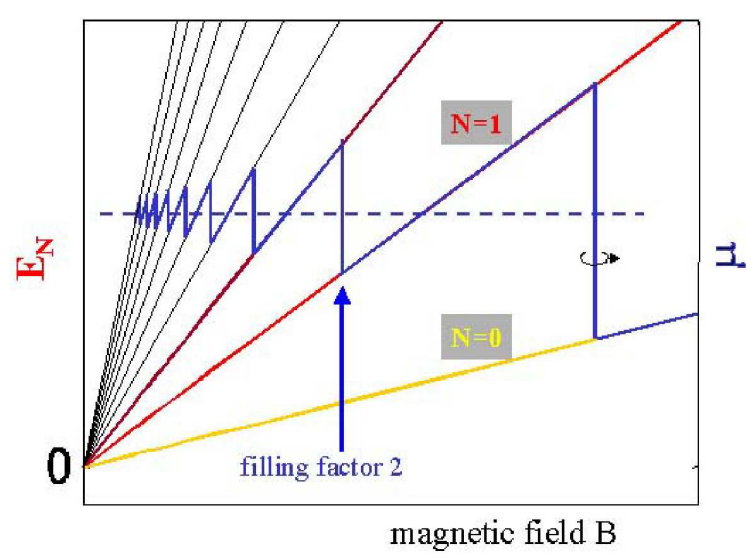

Figure 11: Discrete energy spectrum of a 2DEG in a magnetic field for an ideal system (no spin, no disorder, infinite system, zero temperature). The Fermi energy (full red line) jumps between Landau levels at integer filling factors if the electron concentration is constant.
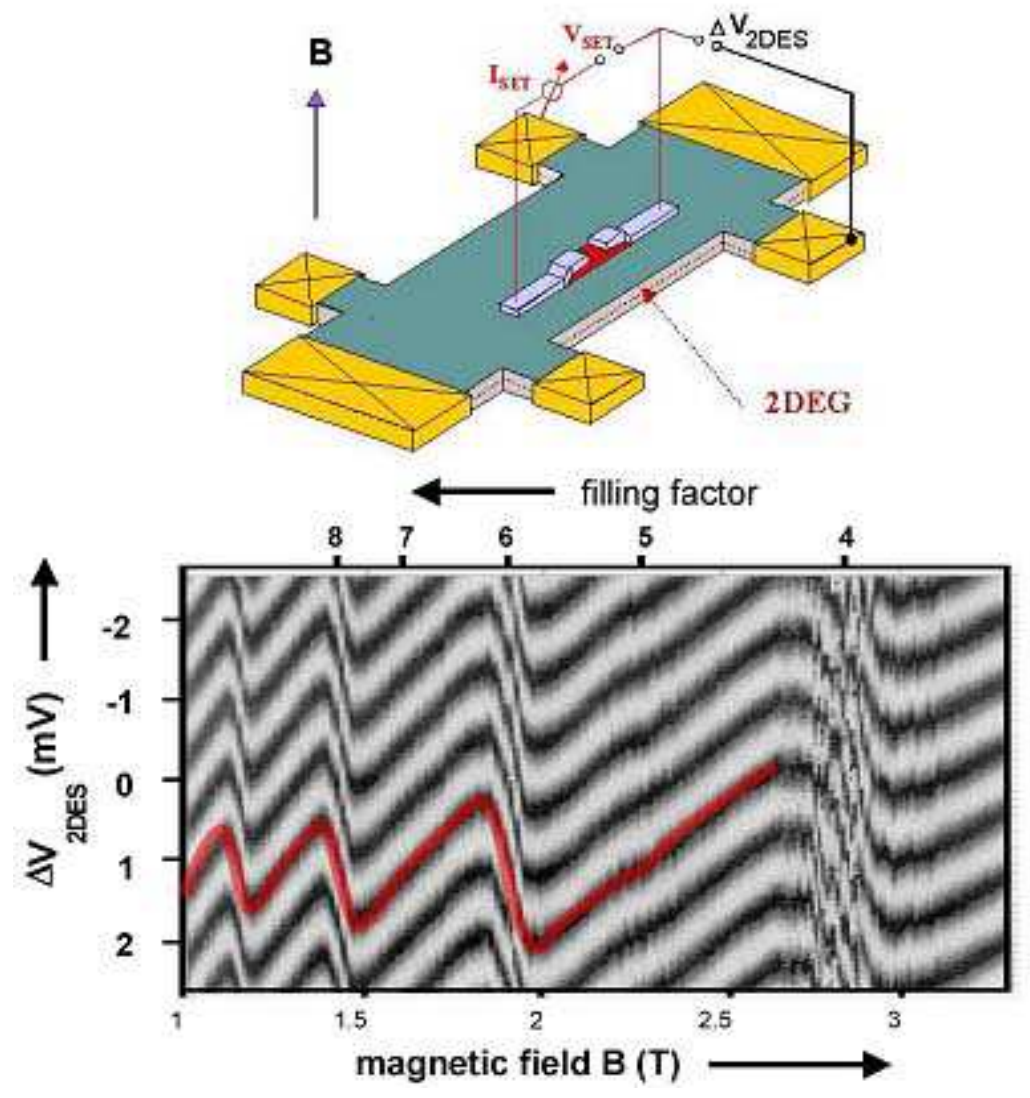

Figure 12: Measured variation of the electrostatic potential of a $2 \mathrm{DEG}$ as a function of the magnetic field. The red line marks a maximum in the coulomb oscillations of a metallic single electron on top of the heterostructure, which corresponds to a constant electrostatic potential of the 2DEG relative to the SET. 
This simple "explanation" of the quantized Hall resistance leads to the correct result but contains unrealistic assumptions. A real Hall device has always a finite width and length with metallic contacts and even high mobility devices contain impurities and potential fluctuations, which lift the degeneracy of the Landau levels. These two important aspects, the finite size and the disorder, will be discussed in the following chapters.

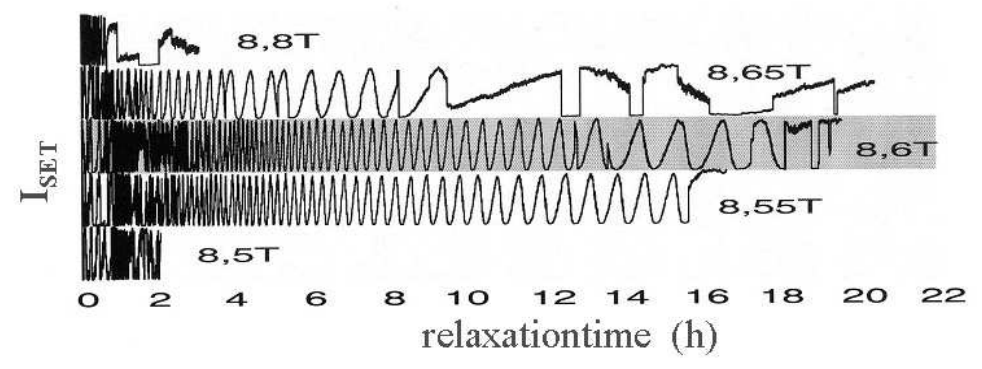

Figure 13: SET current as a function of time for different magnetic fields close to filling factor 1. The oscillations in the current originate from the relaxation of a non-equilibrium electrostatic potential within the 2DEG originating from eddy currents due to the magnetic field sweep in the plateau region. One oscillation in the SET current corresponds to a change in the "gate potential" of about $1 \mathrm{meV}$.

\subsection{Quantum Hall Systems with Disorder}

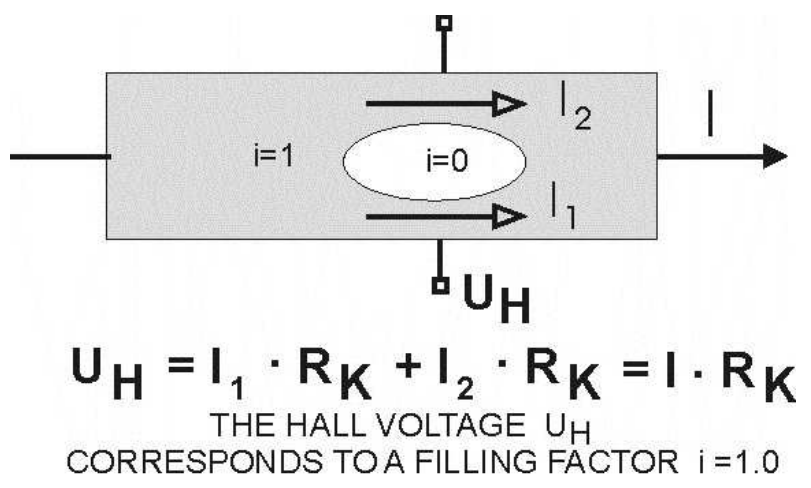

Figure 14: Sketch of a device with a filling factor slightly below 1. Long-range potential fluctuations lead to a finite area within the sample (localized carriers) with vanishing electrons (= filling factor 0 ) surrounded by an equipotential line. The derivation of the measured Hall voltage $U_{H}$ show, that closed areas with another filling factor than the main part of the device leads to a Hall effect which is not influenced by localized electrons.

The experimental fact, that the Hall resistance stays constant even if the filling factor is changed (e.g. by varying the magnetic field at fixed density), cannot be explained within the simple single particle picture for an ideal system. A sketch of the energy spectrum and the Fermi energy as a function of the magnetic field is shown in Fig. 11 for such an ideal system at zero temperature. The Fermi energy (full blue line) is located only at very special magnetic field values in energy gaps between Landau levels so that only at these very special magnetic field values and not in a finite magnetic field range the condition for the observation of the QHE is fulfilled. On the other hand, if one assumes, that the Fermi energy remains constant as a function of the magnetic field (dotted line in Fig. 11), wide plateaus for the quantized Hall resistance are expected, since the Fermi energy remains in energy gaps (which correspond to integer filling factors and therefore 
to quantized Hall resistances) in a wide magnetic field range. However, this picture is unrealistic since one has to assume that the electron concentration changes drastically as a function of the magnetic field. If for example the Fermi energy crosses the Landau level $N=1$, the electron density has to change abruptly by a factor of two from filling factor 2 to filling factor 1 . Such a strong redistribution of charges between the 2DEG and an electron reservoir (doping layers, metallic contacts) are in contradiction with electrostatic calculations. The most direct proof, that the Fermi energy jumps across the gap between Landau levels within a relatively small magnetic field range (at least smaller than the plateau width) is given by measurements of the electrostatic potential between a metal (= wire plus sensor connected to the 2DEG) and the two-dimensional electron gas. The electrochemical potential within the metal - 2DEG system has to be constant (thermodynamic equilibrium) so that the magnetic field dependent variation in the chemical potential (characterized by the Fermi energy) has to be compensated by a change in the electrostatic potential difference between the metallic system and the 2DEG (=contact voltage). Such a variation in the electrostatic potential has been measured directly [28] by using a metallic single electron transistor (SET) at the surface of the quantum Hall device as shown in Fig 12. The electrostatic potential of the 2DEG relative to the SET acts as a gate voltage, which influences drastically the current through the SET. (The SET shows Coulomb blockade oscillations with a gate voltage period of about $1 \mathrm{mV}$ ). The experimental data shown in Fig. 12 clearly demonstrate, that the contact voltage and therefore the chemical potential of the two-dimensional system changes saw-tooth like as expected. The height of the jumps corresponds directly to the energy gap. The "noise" at 2.8 Tesla (filling factor 4) demonstrates, that the gate potential below the SET detector is fluctuating and not fixed by the applied voltage $\Delta V_{2 D E S}$ since the vanishing conductivity in the quantum Hall regime between the metallic contacts at the boundary of the device and the inner parts of the sample leads to floating potentials within the 2DEG system. Recent measurements have demonstrated [29], that time constants of many hours are observed for the equilibration of potential differences between the boundary of a QHE device and the inner part of the sample as shown in Fig. 13. The oscillations in the SET current can be directly translated into a variation of the electrostatic potential below the position of the SET since one period corresponds to a "gate voltage change" for the SET of about $1 \mathrm{meV}$. The non-equilibrium originates from a magnetic field sweep in regions of vanishing energy dissipation, which generates eddy currents around the detector and corresponding Hall potential differences of more than $100 \mathrm{meV}$ perpendicular to these currents. These "Hall voltages" are not measurable at the outer Hall potential probes (at the edge of the sample), since all eddy currents cancel each other. The current distribution is unimportant for the accuracy of the quantized Hall resistance!

In order to explain the width of the Hall plateaus, localized electrons in the tails of broadened Landau levels have to be included. A simple thought experiment illustrates, that localized states added or removed from fully occupied Landau levels do not change the Hall resistance (see Fig. 14). For long range potential fluctuations (e.g. due to impurities located close to the 2DEG) the Landau levels follow this potential landscape so that the energies of the Landau levels change with position within the plane of the device. If the energy separation between Landau levels is larger than the peak value of the potential fluctuation, an energy gap still exists and a fully occupied Landau level (e.g. filling factor 1) with the expected quantized Hall resistance $R_{K}$ can be realized. In this picture, a filling factor 0.9 means, that $10 \%$ of the area of the device (= top of the hills in the potential landscape) becomes unoccupied with electrons as sketched in Fig. 14. The boundary of the unoccupied area is an equipotential line (with an unknown potential) but the externally measured Hall voltage $U_{H}$, which is the sum of the Hall voltages of the upper part of the sample (current $I_{2}$ ) and the lower part (current $I_{1}$ ), adds up to the ideal value expected for the filling factor 1 (grey regions). Eddy currents around the hole with $i=0$ will vary the currents $I_{1}$ and $I_{2}$ but the sum is always identical with the external current $I$.

The quantized Hall resistance breaks down, if electronic states at the Fermi energy are extended across the whole device. This is the case for a half-filled Landau level if the simple percolation picture is applied. Such a singularity at half-filled Landau levels has been observed experimentally [30].

This simple picture of extended and localized electron states indicates, that extended states 


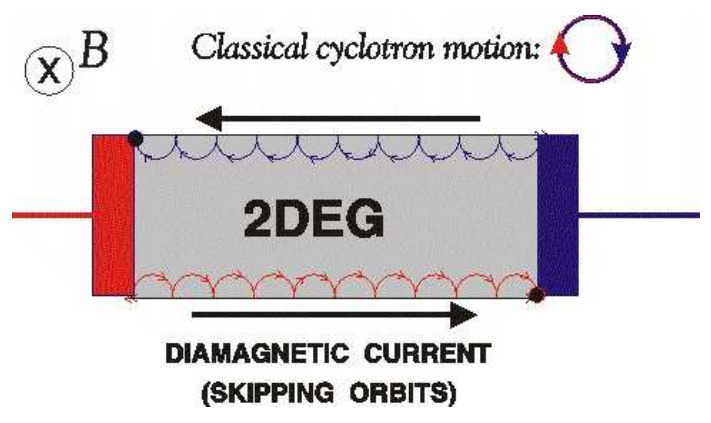

Figure 15: Skipping cyclotron orbits (= diamagnetic current) at the boundaries of a device are equivalent to the edge channels in a 2DEG with finite size.

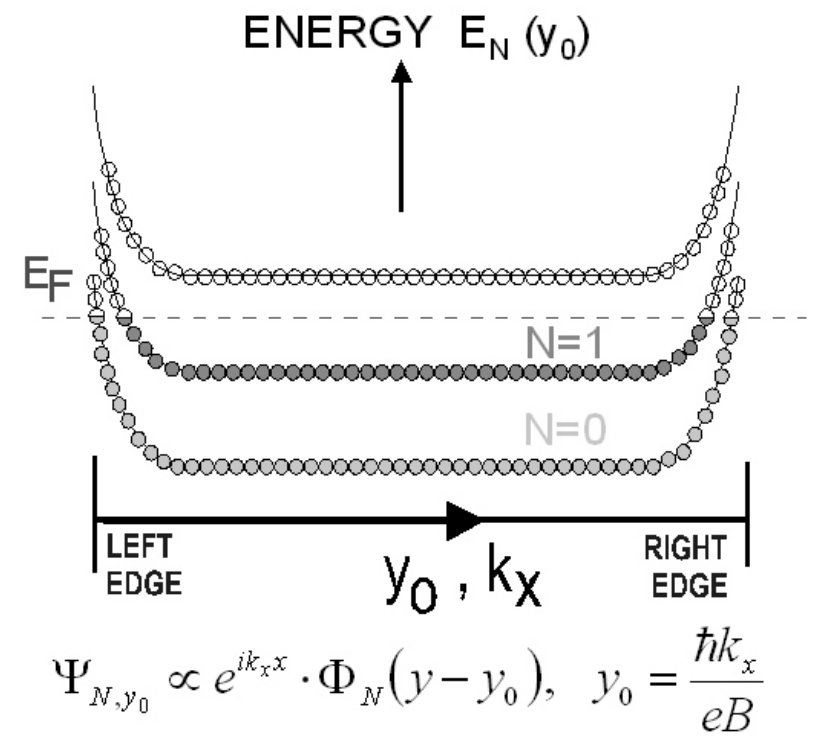

Figure 16: Ideal Landau levels for a device with boundaries. The fully occupied Landau levels in the inner part of the device rise in energy close to the edge forming compressible ("metallic") stripes close to the crossing points of the Landau levels with the Fermi energy $E_{F}$.

always exist at the boundary of the devices. This edge phenomenon is extremely important for a discussion of the quantum Hall effect in real devices and will be discussed in more detail in the next chapter. In a classical picture, skipping orbits as a result of reflected cyclotron orbits at the edge lead to diamagnetic currents as sketched in Fig 15. Therefore, even if the QHE is characterized by a vanishing conductivity $\sigma_{x x}$ (no current in the direction of the electric field), a finite current between source and drain of a Hall device can be established via this diamagnetic current. If the device is connected to source and drain reservoirs with different electrochemical potentials (red and blue color in Fig. 15), the skipping electrons establish different electrochemical potentials at the upper and lower edge respectively. Energy dissipation appears only at the points (black dots in Fig. 15) where the edge potentials differ from the source/drain potentials. 


\subsection{Edge Phenomena in QHE Devices}

The simple explanation of the quantized Hall resistance as a result of fully occupied Landau levels (with a gap at the Fermi energy) breaks down for real devices with finite size. For such a system no energy gap at the Fermi energy exists under quantum Hall condition even if no disorder due to impurities is included. This is illustrated in Fig. 16 where the energy of the Landau levels is plotted across the width of the device. The Fermi energy in the inner part of the sample is assumed to be in the gap for filling factor 2. Close to the edge (within a characteristic depletion length of about $1 \mu \mathrm{m}$ ) the carrier density becomes finally zero. This corresponds to an increase in the Landau level energies at the edge so that these levels become unoccupied outside the sample. All occupied Landau levels inside the sample have to cross the Fermi energy close to the boundary of the device. At these crossing points "half-filled Landau levels" with metallic properties are present.

Selfconsistent calculations for the occupation of the Landau levels show that not lines but metallic stripes with a finite width are formed parallel to the edge [31]. The number of stripes is identical with the number of fully occupied Landau levels in the inner part of the device. These stripes are characterized by a compressible electron gas where the electron concentration can easily be changed since the Landau levels in these regions are only partly filled with electrons and pinned at the Fermi energy. In contrast, the incompressible stripes between the compressible regions represent fully occupied Landau levels with the typical isolating behavior of a quantum Hall state. If the single electron transistor shown in Fig. 12 is located above an incompressible region, increased noise is observed in the SET current similar to the noise at 2.8 Tesla in Fig. 12. In order to visualize the position of the stripes close to the edge, not the SET is moved but an artificial edge (= zero carrier density) is formed with a negative voltage at a gate metal located close to the SET. The edge (= position with vanishing carrier density) moves with increasing negative gate voltage closer to the detector and alternatively incompressible (increased SET noise) and compressible strips (no SET noise) are located below the SET detector. The experimental results in Fig. 17 confirm qualitatively the picture of incompressible stripes close to the edge which move away from the edge with increasing magnetic field until the whole inner part of the device becomes an incompressible region at integer filling factor. At slightly higher magnetic fields this "bulk" incompressible region disappears and only incompressible stripes with lower filling factor remain close to the boundary of the device.

The influence of the incompressible stripes on the Hall potential distribution has been measured with an atomic force microscope. The results in Fig. 18 show, that the innermost incompressible stripe has such strong "insulating" properties that about $50 \%$ of the Hall potential drops across this stripe. The other $50 \%$ of the Hall potential drops close to the opposite boundary of the device across the equivalent incompressible stripe. In principle the incompressible stripes should be able to suppress the backscattering across the width of a Hall device so that even for an ideal device without impurities (= localized states due to potential fluctuations) a finite magnetic field range with vanishing resistivity and quantized Hall plateaus should exist [32]. However, the fact, that the plateau width increases systematically with increasing impurity concentration (and shows the expected different behavior for attractive and repulsive impurities) shows, that localized states due to impurities are the main origin for the stabilization of the quantized Hall resistance within a finite range of filling factors. A vanishing longitudinal resistivity always indicates, that a backscattering is not measurable. Under this condition the quantized Hall resistance is a direct consequence of the transmission of one-dimensional channels [33].

\section{Correlated Electron Phenomena in Quantum Hall Systems}

Many physical properties of the quantum Hall effect can be discussed in a single electron picture but it is obvious that the majority of modern research and publications in this field include electron correlation phenomena. Even if the fractional quantum Hall effect [34] (which is a manifestation of the strong electron-electron interaction in a two-dimensional system) can be nicely discussed as the integer quantum Hall effect of weakly interacting quasi particles called composite fermions [35], the many-body wave function of the quantum Hall system is the basis for the discussion of different exciting new phenomena observed in correlated two-dimensional systems in strong magnetic fields. 
Many of these new phenomena can only be observed in devices with extremely high mobility where the electron-electron interaction is not destroyed by disorder. Experiments on such devices indicate, that phenomena like superfluidity and Bose-Einstein condensation [36], skyrmionic excitations [37], fractional charges [38], a new zero resistance state under microwave radiation [39] or new phases based on a decomposition of a half-integer filling factor into stripes and bubbles with integer filling factors [40] are observable. The two-dimensional electron system in strong magnetic field seems to be the ideal system to study electron correlation phenomena in solids with the possibility to control and vary many parameters so that the quantum Hall effect will remain a modern research field also in the future.

\section{References}

[1] T. Ando, A. Fowler, and F. Stern, Rev. Mod. Phys. 54, 437 (1982).

[2] A.B. Fowler, F.F. Fang, W.E. Howard, and P.J. Stiles, Phys Rev. Lett. 16, 901 (1966).

[3] H. Aoki and H. Kamimura, Solid State Comm. 21, 45 (1977).

[4] Th. Englert and K. v. Klitzing, Surf. Sci. 73, 70 (1978).

[5] K. v. Klitzing, G. Dorda and M. Pepper, Phys. Rev. Lett. 45, 494 (1980).

[6] Y.S. Myung, Phys. Rev. D 59, 044028 (1999).

[7] M. Greiter, Int. Journal of Modern Physics A 13 (8), 1293 (1998).

[8] S.C. Zhang and J. Hu, Science 294, 823 (2001).

[9] V. Privman, I.D. Vagner and G. Kventsel, Phys. Lett. A239, 141 (1998).

[10] M. Fabinger, J. High Energy Physics 5, 37 (2002).

[11] F.W. Hehl, Y.N. Obukhov, and B. Rosenow, Phys. Rev. Lett. 93, 096804 (2004).

[12] R.E. Prange and S. Girvin (ed.), The Quantum Hall Effect, Springer, New York (1987).

[13] M. Stone (ed.), Quantum Hall Effect, World Scientific, Singapore (1992).

[14] M. Janen, O. Viehweger, U. Fastenrath, and J. Hajdu, Introduction to the Theory of the Integer Quantum Hall Effect, VCH Weinheim (1994).

[15] T. Chakraborty, P. Pietilinen, The Quatnum Hall Effects, 2nd Ed., Springer, Berlin (1995).

[16] S. Das Sarma and A. Pinczuk, Perspectives in Quantum Hall Effects, John Wiley, New York (1997).

[17] O. Heinonen (ed.), Composite Fermions: A Unified View of the Quantum Hall Regime, World Scientific, Singapore (1998).

[18] Z.F. Ezawa, Quantum Hall Effects - Field Theoretical Approach and Related Topics, World Scientific, Singapore (2002).

[19] D. Yoshioka,Quantum Hall Effect, Springer, Berlin (2002).

[20] Proc. Int. Symp. Quantum Hall Effect: Past, Present and Future, Editor R. Haug and D. Weiss, Physica E20 (2003).

[21] A.M. Thompson and D.G. Lampard, Nature 177, 888 (1956).

[22] T.J. Quinn, Metrologia 26, 69 (1989).

[23] F. Delahaye, T.J. Witt, R.E. Elmquist and R.F. Dziuba, Metrologia 37, 173 (2000). 
[24] F. Delahaye and B. Jeckelmann, Metrologia 40, 217 (2003).

[25] The most recent values of fundamental constants recommended by CODATA are available at: http://physics.nist.gov/cuu/constants/index.html.

[26] E.R. Williams, R.L. Steiner, D.B. Nevell, and P.T. Olsen, Phys. Rev. Lett. 81, 2404 (1998).

[27] E.H. Hall, American Journal of Mathematics, vol ii, 287 (1879).

[28] Y.Y. Wei, J. Weis, K. v. Klitzing and K. Eberl, Appl. Phys. Lett. 71 (17), 2514 (1997).

[29] J. Hüls, J. Weis, J. Smet, and K. v. Klitzing, Phys. Rev. B69 (8), 05319 (2004).

[30] S. Koch, R.J. Haug, K. v. Klitzing, and K. Ploog, Phys. Rev B43 (8), 6828 (1991).

[31] D.B.Chklovskii, B.I. Shklovskii, and L.I. Glazmann, Phys. Rev. B46, 4026 (1999).

[32] A. Siddiki and R.R. Gerhardts, Phys. Rev. B70, (2004).

[33] M. Bttiker, Phys. Rev. B38, 9375 (1988).

[34] D.C. Tsui, H.L. Stormer, and A.C. Crossard, Phys. Rev. Lett. 48, 1559 (1982).

[35] J.K. Jain, Phys. Rev. Lett. 63, 199 (1989).

[36] M.Kellogg, J.P. Eisenstein, L.N. Pfeiffer, and K.W. West, Phys. Rev. Lett. 93, 036801 (2004).

[37] A.H. MacDonald, H.A. Fertig, and Luis Brey, Phys. Rev. Lett. 76, 2153 (1996).

[38] R. de-Picciotto, M. Reznikov, M. Heiblum, Nature 389, 162 (1997).

[39] R.G. Mani, J.H. Smet, K. V. Klitzing, V. Narayanamurti, W.B. Johnson, and V. Urmanski, Nature 420, 646 (2002).

[40] M.P. Lilly, K.B. Cooper, J.P. Eisenstein, L.N. Pfeiffer, and K.W. West, Phys. Rev. Lett. 82, 394 (1999). 